\title{
Radiation furnace for synchrotron dark-field X-ray microscopy experiments
}

Yildirim, C.; Vitoux, H.; Dresselhaus-Marais, L. E.; Steinmann, R.; Watier, Y.; Cook, P. K.; Kutsal, M.; Detlefs, C.

Published in:

Review of Scientific Instruments

Link to article, DOI:

$10.1063 / 1.5141139$

Publication date:

2020

Document Version

Publisher's PDF, also known as Version of record

Link back to DTU Orbit

Citation (APA):

Yildirim, C., Vitoux, H., Dresselhaus-Marais, L. E., Steinmann, R., Watier, Y., Cook, P. K., Kutsal, M., \& Detlefs, C. (2020). Radiation furnace for synchrotron dark-field X-ray microscopy experiments. Review of Scientific Instruments, 91(6), [065109]. https://doi.org/10.1063/1.5141139

\section{General rights}

Copyright and moral rights for the publications made accessible in the public portal are retained by the authors and/or other copyright owners and it is a condition of accessing publications that users recognise and abide by the legal requirements associated with these rights.

- Users may download and print one copy of any publication from the public portal for the purpose of private study or research.

- You may not further distribute the material or use it for any profit-making activity or commercial gain

- You may freely distribute the URL identifying the publication in the public portal

If you believe that this document breaches copyright please contact us providing details, and we will remove access to the work immediately and investigate your claim 


\section{Radiation furnace for synchrotron dark- field x-ray microscopy experiments}

Cite as: Rev. Sci. Instrum. 91, 065109 (2020); https://doi.org/10.1063/1.5141139

Submitted: 05 December 2019 . Accepted: 23 May 2020 . Published Online: 12 June 2020

(D) C. Yildirim, H. Vitoux, (D) L. E. Dresselhaus-Marais, R. Steinmann, Y. Watier, P. K. Cook, (D) M. Kutsal, and (D) C. Detlefs

View Online

\section{ARTICLES YOU MAY BE INTERESTED IN}

Imaging microstructural dynamics and strain fields in electro-active materials in situ with dark field x-ray microscopy

Review of Scientific Instruments 91, 065103 (2020); https://doi.org/10.1063/1.5142319

Improving the angle measurement accuracy of circular grating

Review of Scientific Instruments 91, 065108 (2020); https://doi.org/10.1063/5.0001660

A novel gradient composition spreading and nanolayer stacking process for combinatorial thin-film materials library fabrication

Review of Scientific Instruments 91, 065107 (2020); https://doi.org/10.1063/5.0011119

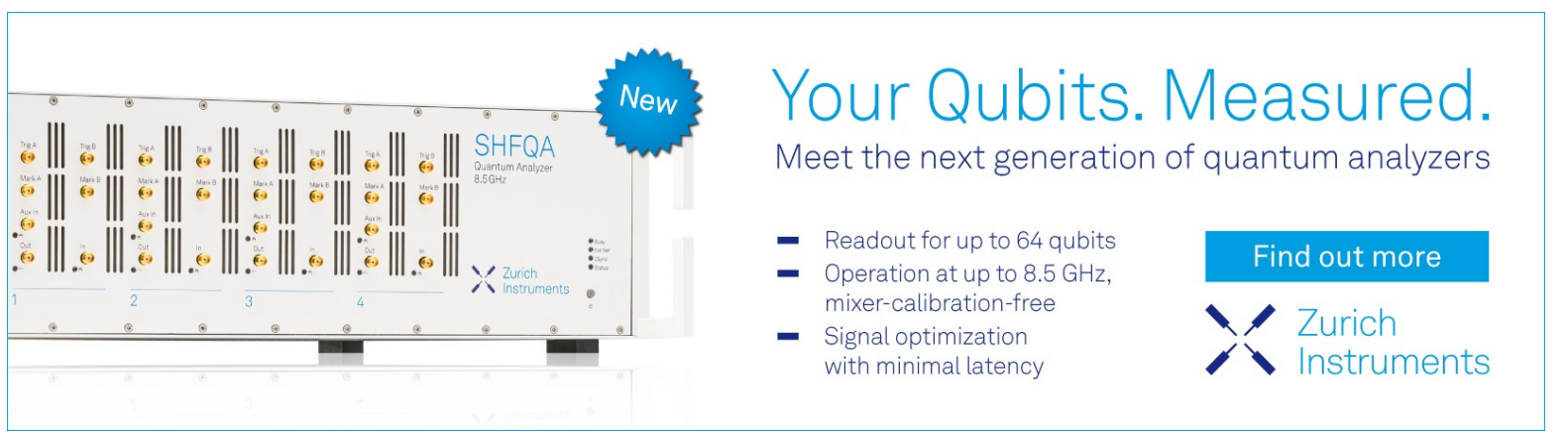




\title{
Radiation furnace for synchrotron dark-field x-ray microscopy experiments
}

\author{
Cite as: Rev. Sci. Instrum. 91, 065109 (2020); doi: 10.1063/1.5141139 \\ Submitted: 5 December 2019 • Accepted: 23 May 2020 • \\ Published Online: 12 June 2020
}

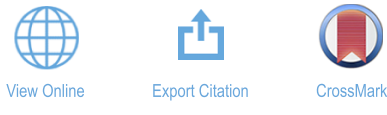

\author{
C. Yildirim, ${ }^{1,2, a)}$ (D) H. Vitoux, ${ }^{1}$ L. E. Dresselhaus-Marais, ${ }^{3}$ (D) R. Steinmann, ${ }^{1, b)}$ Y. Watier, ${ }^{1}$ P. K. Cook, ${ }^{1, c)}$ M. Kutsal, \\ and C. Detlefs
}

\author{
AFFILIATIONS \\ ${ }^{1}$ Experiments Division, European Synchrotron Radiation Facility, 71 Avenue des Martyrs, CS40220, \\ 38043 Grenoble Cedex 9, France \\ ${ }^{2}$ OCAS, Pres. J.F. Kennedylaan 3, BE-9060 Zelzate, Belgium \\ ${ }^{3}$ Lawrence Livermore National Laboratory, Physics and Life Sciences, Physics Division, 7000 East Avenue, L-487, \\ Livermore, California 94550, USA \\ ${ }^{4}$ Department of Physics, Technical University of Denmark, 2800 Kgs. Lyngby, Denmark
}

\begin{abstract}
a) Author to whom correspondence should be addressed: can.yildirim@esrf.fr. Present address: LETI, CEA, 17 avenue des Martyrs, 38054 Grenoble, France.

b) Present address: AS Special Devices, 8 avenue Victor Hugo, 38130 Echirolles, France.

c) Present address: Institut für Physik und Materialwissenschaft (IPM), University of Natural Resources and Life Sciences, Peter-Jordan-Straße 82, 1190 Vienna, Austria.
\end{abstract}

\begin{abstract}
We present a multi-purpose radiation furnace designed for x-ray experiments at synchrotrons. The furnace is optimized specifically for darkfield x-ray microscopy (DFXM) of crystalline materials at beamline ID06 of the European Synchrotron Radiation Facility. The furnace can reach temperatures above $1200^{\circ} \mathrm{C}$ with a thermal stability better than $10^{\circ} \mathrm{C}$, with heating and cooling rates up to $30 \mathrm{~K} / \mathrm{s}$. The non-contact heating design enables samples to be heated either in air or in a controlled atmosphere contained within a capillary tube. The temperature was calibrated via the thermal expansion of an $\alpha$-iron grain. Temperature profiles in the $y$ and $z$ axes were measured by scanning a thermocouple through the focal spot of the radiation furnace. In the current configuration of the beamline, this furnace can be used for DFXM, near-field xray topography, bright-field $\mathrm{x}$-ray nanotomography, high-resolution reciprocal space mapping, and limited powder diffraction experiments. As a first application, we present a DFXM case study on isothermal heating of a commercially pure single crystal of aluminum.
\end{abstract}

Published under license by AIP Publishing. https://doi.org/10.1063/1.5141139

\section{INTRODUCTION}

The microstructure of a material often governs its performance in engineering applications. Heat treatments are used in industry to produce the desired mechanical properties in metals and alloys, as the microstructure depends on the material's thermal history. ${ }^{1}$ Indeed, heat treatments are used to change the microstructure of a host of different materials, including ceramics and optical materials, to improve the material's performance for diverse applications, including aerospace, electronics, communication, and medicine. To understand how temperature drives the microstructural changes in materials, this wide range of applications requires in situ studies to understand the microscopic processes during heat treatment to refine designs and develop new engineering materials.

Many industrial materials have hierarchical structures, with grains and domains that span several length scales; hightemperature studies of these materials thus require non-destructive measurements that can accurately measure structures over a range of $10 \mathrm{~nm}$ to $1 \mathrm{~mm}$ with a high angular resolution. Electronbased microscopy methods such as electron back-scatter diffraction (EBSD) and transmission electron microscopy (TEM) can probe several length scales with a high spatial resolution. However, the low penetration depth of electron beams limits these measurements to surfaces or very thin samples (often including invasive sectioning processes). ${ }^{2}$ Synchrotron diffraction methods such as 
3DXRD ${ }^{3}$ and its near-field derivative, diffraction contrast tomography (DCT), ${ }^{4}$ can measure 3D information in a non-destructive manner. However, these tools cannot map highly deformed samples or $\mathrm{nm}$-sized domains because of overlapping peaks and insufficient spatial resolution, respectively.

Dark-field $\mathrm{x}$-ray microscopy (DFXM) is a new technique that non-destructively collects three-dimensional (3D) information about the material's strain and orientation at different length scales. ${ }^{5-9}$ Analogous to dark-field electron microscopy, DFXM uses an objective lens to magnify features in a crystalline sample that diffract. Using high-energy synchrotron $\mathrm{x}$ rays, the beam penetrates the crystal, resolving deeply embedded features. Full 3D mapping can be performed in minutes, with changes to the field of view and spatial resolution requiring only simple reconfiguration of the $\mathrm{x}$-ray objective lens. The spatial resolution has reached $100 \mathrm{~nm}$ with an angular resolution of $0.001^{\circ}$. The high resolution and high sensitivity coupled with the deep penetration depth makes DFXM a unique tool to study the in situ behavior of crystalline engineering materials at high temperatures.

Many different types of heating systems are used for synchrotron experiments, including resistive heating, ${ }^{10,11}$ laser heating, ${ }^{12,13}$ inductive heating, ${ }^{14}$ and radiation furnaces. ${ }^{15-17}$ In radiation furnaces (also called optical or mirror furnaces), reflective mirrors focus radiation from a lamp (e.g., halogen) onto the sample. This design makes radiation furnaces compact, with high heating and cooling rates and high thermal stability for experiments in both transmission and reflection geometries. Most existing radiation furnace designs have been developed for in situ x-ray diffraction (XRD) studies; however, the aforementioned advantages also present advantages for in situ diffraction imaging studies, which also require a compact design and high thermal stability. Here, we present a non-contact radiation furnace, designed specifically to be compatible with DFXM experiments at the European Synchrotron Radiation Facility (ESRF)

\section{SPECIFICATIONS}

The initial goals of this furnace focused on metals, so we focus the calibrations on the temperature range relevant to process steel, namely, from room temperature to above $1200^{\circ} \mathrm{C}$. Within this range, we also explored fast rates of heating and cooling, which are important for industrial applications that require isothermal studies and additive manufacturing. Cooling rates of $25-30 \mathrm{~K} / \mathrm{s}$ are typical for austenite to pearlite and martensite transition in many steel alloys. ${ }^{18}$ Therefore, the furnace is needed to achieve these cooling rates or more.

A well-defined thermal stability is essential to interpret the material's response to temperature. The microstructure of a material (especially ductile metals) is also very sensitive to additional stresses from thermal fluctuations, which can produce results that are not representative of an isothermal material. This means that temperature fluctuations must be avoided such that they do not alter the state of the sample during a DFXM scan for isothermal studies. As DFXM experiments use objective lenses that have small effective apertures, they are highly sensitive to changes in the diffraction angle (varies with the $d$-spacing) that originate from thermal expansion or strain.

In addition to the primary application, DFXM, the furnace also needs to be compatible with the other x-ray techniques available at ID06, including bright-field tomography, near-field diffraction topography, and high-resolution reciprocal space mapping. For use over this wide range of experimental capabilities, the furnace thus needs to have clear apertures for the incident, transmitted, and diffracted $\mathrm{x}$-ray beams with scattering angles up to $2 \theta \approx 30^{\circ}$ in the vertical scattering plane (i.e., the maximum scattering angle of the current instrument on ID06). ${ }^{8,9}$ Finally, the furnace needs to permit a full $360^{\circ}$ sample rotation about the $y$ axis to allow experiments to rock the crystal along one rotational axis for diffraction in the vertical geometry. ${ }^{8}$ At the same time, the apertures need to be as small as possible to prevent heat from leaking out of the system.

As DFXM images embedded crystalline regions beneath the material's surface, the x-ray measurements are performed in transmission (Laue) geometry, with photon energies covering $15-35 \mathrm{keV}$. Typical samples have a size of $0.5-2 \mathrm{~mm}$ in the $x$ and $z$ directions with a length of several $\mathrm{mm}$ along the $y$ axis. The samples are mounted inside a quartz capillary that can be filled with either air or an inert atmosphere.

The primary design constraint for the furnace was the limited space available on the sample stage at ID06, ${ }^{9}$ specifically, the distance between the sample mounting surface and the goniometer's center of rotation (i.e., the sample position). The sample mounting surface of the goniometer is on the negative $y$ side [right side in Fig. 1(b)]. Furthermore, the furnace needs to be small enough to allow a near-field camera to be positioned $50-100 \mathrm{~mm}$ downstream from the sample for alignment or imaging experiments (e.g., near-field $\mathrm{x}$-ray topography).
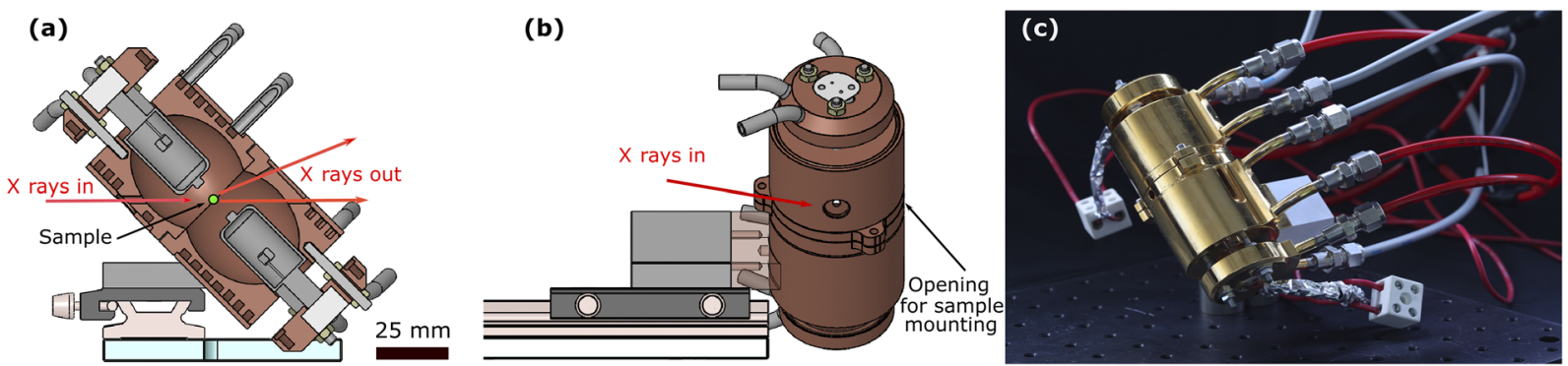

FIG. 1. General layout of the furnace: (a) side view and (b) front view. The incident beam travels along the $x$-axis, the $y$-axis is to the left in (b), and the $z$-axis is up. (c) Photograph of the radiation furnace. The opening for mounting the sample is on the left and the slot for the scattered beam is on the right. 


\section{IMPLEMENTATION}

To accommodate the specifications and constraints listed above, we constructed a non-contact radiation furnace, where the furnace and sample are mounted independently. This has several benefits:

- Heat transfer from the furnace to the goniometer is reduced, thus minimizing long-timescale thermal drift of the sample position.

- The furnace and the associated power cables and cooling water lines do not increase the mechanical load on the goniometer stages.

- Potential vibrations of the furnace (e.g., due to water cooling) are not transmitted to the sample.

- The furnace remains stationary during $\mathrm{x}$-ray experiments even when the sample is rotated or moved, minimizing the necessary size of the openings for the incident and diffracted beam. This reduces power loss, increasing the efficiency of the furnace and minimizing inhomogeneities in the heating.

- As the furnace is mounted on a separate motorized $y$ translation stage, the furnace may be retracted without repositioning the sample. This can be helpful to access a wider range of scattering angles, as has been performed in interrupted annealing measurements with 3DXRD.

- The sample's temperature can be changed rapidly, as only the lamps and sample are "hot" (i.e., the thermal inertia of the system is very small). In principle, the rapid temperature response makes proportional integral derivative (PID) regulation easy.

A schematic layout of the furnace is shown in Fig. 1. Two lamps are used as the heating elements in this design, as preliminary tests with a single bulb did not reach the desired temperatures. The bulbs are positioned in a reflective cavity with a shape defined by two intersecting ellipsoids. The sample is positioned near the shared central focus, and the bulbs sit at the other two foci, as shown in Fig. 1. France.

The furnace was constructed by AS Special Devices, Echirolles,

The reflectors were machined from copper, then polished, and gold plated. Integrated cooling channels regulate the temperature in the furnace, circulating either air or water. Before machining, the copper casing was annealed at $850^{\circ} \mathrm{C}$ for $12 \mathrm{~h}$ to relieve any built-up stresses in the material, preventing the material from deforming as the outer shell and water connections are brazed in the subsequent machining.

Machining of the furnace was carried out in two steps. First, the internal shape was machined to the rough-shape of an ellipsoid, with axes $1 \mathrm{~mm}$ smaller than the desired shape. The outer shell and water connections were then brazed, and the ellipsoid was subsequently machined to its final size and shape.

The cavities were then electropolished, refining the surface roughness to $R_{a} \approx 0.1 \mu \mathrm{m}$. Loss of material during the electropolishing was partially compensated by a surface layer of nickel, which prevents diffusion between the copper casing and the gold layer that was deposited on the surface.

The integrated cooling channels keep the furnace's outer surface below $20^{\circ} \mathrm{C}$, even when the sample is heated to internal temperatures above $1000^{\circ} \mathrm{C}$.
The sizes and flow rates for the cooling channels were calculated to allow for locations for the input and output feeds that could minimize forces on the furnace as much as possible. Ultimately, reducing the net force would mitigate vibrations from the water circulation that could reduce blur of the experiment's images.

Parasitic heating of the surroundings is thus limited to blackbody radiation that escapes through apertures for the $\mathrm{x}$-ray beams, the sample, and near the bases of the two lamps. The cooling channels allow for safe operation of equipment surrounding the furnace, including the goniometer, the sample stage, and the near-field camera.

The sample is positioned $3 \mathrm{~mm}$ upstream of the furnace radiations' focal spot. This gives an illuminated circular area with a diameter of $5 \mathrm{~mm}$ through the depth of the sample, which heats the sample more homogeneously than it would if the sample were in the exactly focal spot. The defocused radiation therefore improves the precision of the temperature with respect to possible misalignment of the sample's position in the furnace.

The casing's apertures for its heat lamps were designed to reduce air convection at the sample position and-as much as possible-the resulting temperature inhomogeneities that they could also generate around the sample. Its diameter was optimized using a flow simulation program in order that $75 \%$ of the air enters through the lower lamp's aperture and exits through the upper lamp's aperture.

The heating elements in the furnace are two identical $400 \mathrm{~W}$ halogen light bulbs. The lamps are connected in parallel to a Delta Elektronika DC power supply, operated at constant power via a software feedback loop. The required power for a given set-point temperature was obtained from a lookup table calculated from calibration measurements with a thermocouple at the sample position. The beamline control software can control the sample's temperature, and thus, data can be acquired automatically with slow, step-wise temperature ramps or interrupted annealing over a precise duration. Different samples may require a different power to achieve the same set-point temperature, based on the surface reflectivity and absorption cross section over the radiation furnace's energy spectrum.

Samples can be inserted horizontally (perpendicular to the scattering plane) through an opening with $\approx 5 \mathrm{~mm}$ diameter. The distance from the outside edge of the furnace to the sample position is $\approx 27 \mathrm{~mm}$. The size of this opening allows the samples to be sealed in capillaries that are backfilled with a desired atmosphere and, if necessary, mounted to a Huber pin with a temperature-resistant ceramic glue to ensure stability. The aperture for the incident $\mathrm{x}$ rays has diameter of $2.5 \mathrm{~mm}$, which matches the field of view of the near-field camera. On the downstream side, a vertical slot with $2.5 \mathrm{~mm}$ width allows $\mathrm{x}$-ray beams with vertical scattering angles of up to $30^{\circ}$ to exit the furnace unobstructed. The blackbody radiation within the furnace is focused by double-ellipsoid mirrors. The axis or rotation of the double-ellipsoid is inclined $45^{\circ}$ toward the incident $\mathrm{x}$-ray beam, which maximizes the accessible range of scattering angles, as shown in Fig. 1.

There are some drawbacks of the chosen design geometry:

- The radiation's focal point within the furnace is located $27 \mathrm{~mm}$ from the outer edges of the furnace (along the radius of the cylinder). The sample support must, therefore, be long 
and thin to position the sample at the center of the furnace, with supporting material that is also heated by the blackbody radiation. Temperature changes and thermal expansion within the supporting materials may require adjustments to the sample position after large changes in temperature. The support should therefore be made from a material with low thermal expansion (e.g., quartz) and ideally with a high infrared reflectively or low infrared absorption.

- The small diameter of the sample aperture $(5 \mathrm{~mm})$ strongly limits the range of sample rotations about the $x$ (incident beam) and $z$ (vertical) axes. Increasing the accessible angular range would require a larger lateral opening in the furnace, which would leak significantly more heat onto the goniometer, potentially destabilizing the thermal uniformity at the sample position. Furthermore, these changes could also cause asymmetry and inhomogeneity in the radiation and result in temperature gradients across the sample.

- The sample's temperature is not measured directly. The equilibrium sample temperature depends on a combination of parameters, including the sample's absorption cross section for the relevant blackbody radiation spectrum and its emissivity. ${ }^{20}$ At a given power, a strongly absorbing and lowemissivity sample may be much hotter than a highly reflective or transmissive sample. This means that the relationship between the power to the lamps and the temperature at the sample must be independently calibrated using, for example, thermal expansion measurements.

- The small mass of heated samples (thermal mass) and the capability for rapid temperature changes both reduce the system's short-term temperature stability.

\section{RESULTS AND DISCUSSIONS}

\section{A. Characterization by thermocouple}

Figure 2 shows the temperature response of a K-type thermocouple located at the center of the furnace at different power values. We used a fitting function that contains the sum of two exponential functions to describe the time-temperature behavior of the furnace,

$$
T=T_{0}+A_{\text {slow }} \exp \left(-t / \tau_{\text {slow }}\right)+A_{\text {fast }} \exp \left(-t / \tau_{\text {fast }}\right) .
$$

The results of the fits are reported in Table I. For the first temperature step, we compute the thermal stability at $5 \mathrm{~min}$ to be $4.63 \mathrm{~K}$ based on the exponential fit, which yields $\tau_{\text {slow }}=137 \pm 6.7 \mathrm{~s}$ with an amplitude of $A_{\text {slow }}=-41 \pm 0.6 \mathrm{~K}$. For the fourth temperature step, which cools the sample to room temperature and, as radiative heat transfer becomes considerably small when the shell and the sample are nearly at room temperature, is therefore the slowest of these steps, the thermal stability at $5 \mathrm{~min}$ is calculated as $7.72 \mathrm{~K}$ from the fit (Table I).

We note that the two exponentials in each fit correspond to two different stages for each heating step, each with their own inherent timescales. We interpret the fast heating stages to be equilibration between the furnace and the sample, while the slow heating stage corresponds to the equilibration of the sample holder to the new temperature (in this case, the stainless steel housing of the thermocouple). The diffraction-based calibration measurements used a

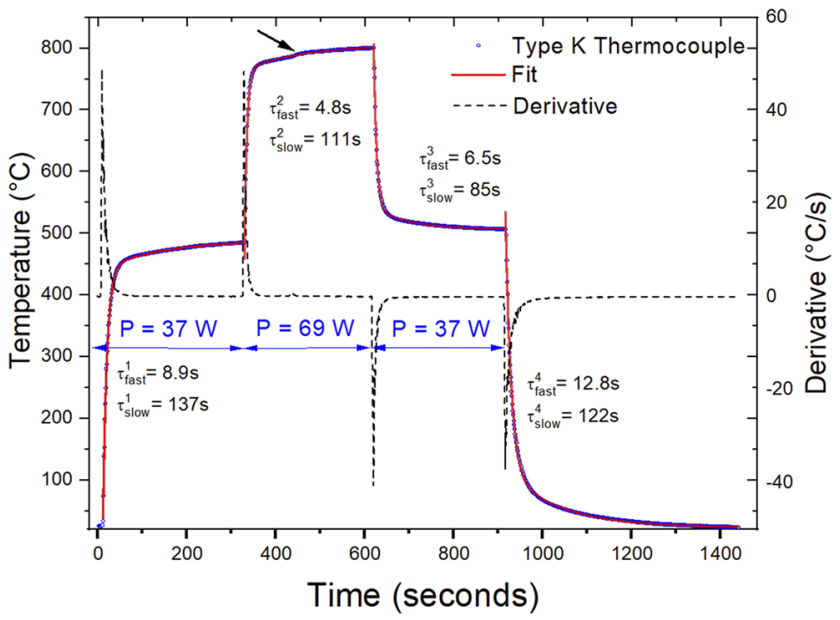

FIG. 2. Temperature calibration using a K-type thermocouple showing heating and cooling rates for given set temperatures.

quartz capillary as the sample holder and showed better thermal stability after $5 \mathrm{~min}$, indicating less lag time from the slower process. Further improvements to the timescales of thermal equilibration and stability could be made by implementing a closed-loop feedback system.

We also note that the measured temperatures are different at the two plateaus of $P=37 \mathrm{~W}$. This result is caused by the blackening of the thermocouple (shown by the black arrow in Fig. 2) during the heating at $P=69 \mathrm{~W}$, which increases its absorption efficiency for the furnace's radiation and therefore increases the temperature reached the second time when the thermocouple was heated with $P=37 \mathrm{~W}$.

The heating and cooling rates were calculated by fitting the temperature-time plots using exponential functions, as shown in Fig. 2. Heating to a set temperature always results in a faster rate than that of the corresponding temperature decrease, as there is no active cooling element to drive the cooling rates faster than thermal diffusion of the sample. The cooling rates could be increased by blowing room temperature air or nitrogen gas through the cavity of the furnace, although this would also introduce thermal gradients, as it would be a surface-driven cooling element. Note that in this experiment, the internal copper elements of the furnace were cooled.

Figures 3(a) and 3(b) show the spatial profile of temperature along the $y$ and $z$ directions for different lamp powers, as measured

TABLE I. Fit parameters of the temperature calibration using the K-type thermocouple.

\begin{tabular}{lcccr}
$\begin{array}{l}\text { Temperature } \\
\text { step }\end{array}$ & $\mathrm{A}_{\text {fast }}(\mathrm{K})$ & \multicolumn{1}{c}{$\tau_{\text {fast }}(\mathrm{s})$} & \multicolumn{1}{c}{$\mathrm{A}_{\text {slow }}(\mathrm{K})$} & \multicolumn{1}{c}{$\tau_{\text {slow }}(\mathrm{s})$} \\
\hline 1 & $-78.6 \pm 0.8$ & $8.91 \pm 0.05$ & $-41 \pm 0.5$ & $137.6 \pm 6.7$ \\
2 & $-264.6 \pm 0.9$ & $4.80 \pm 0.03$ & $-40.9 \pm 0.34$ & $111.1 \pm 3.4$ \\
3 & $-298.4 \pm 1.5$ & $6.53 \pm 0.06$ & $33.7 \pm 0.7$ & $85.2 \pm 4.2$ \\
4 & $419.9 \pm 1.6$ & $12.8 \pm 0.1$ & $90.3 \pm 2.5$ & $122.1 \pm 2.5$ \\
\hline
\end{tabular}



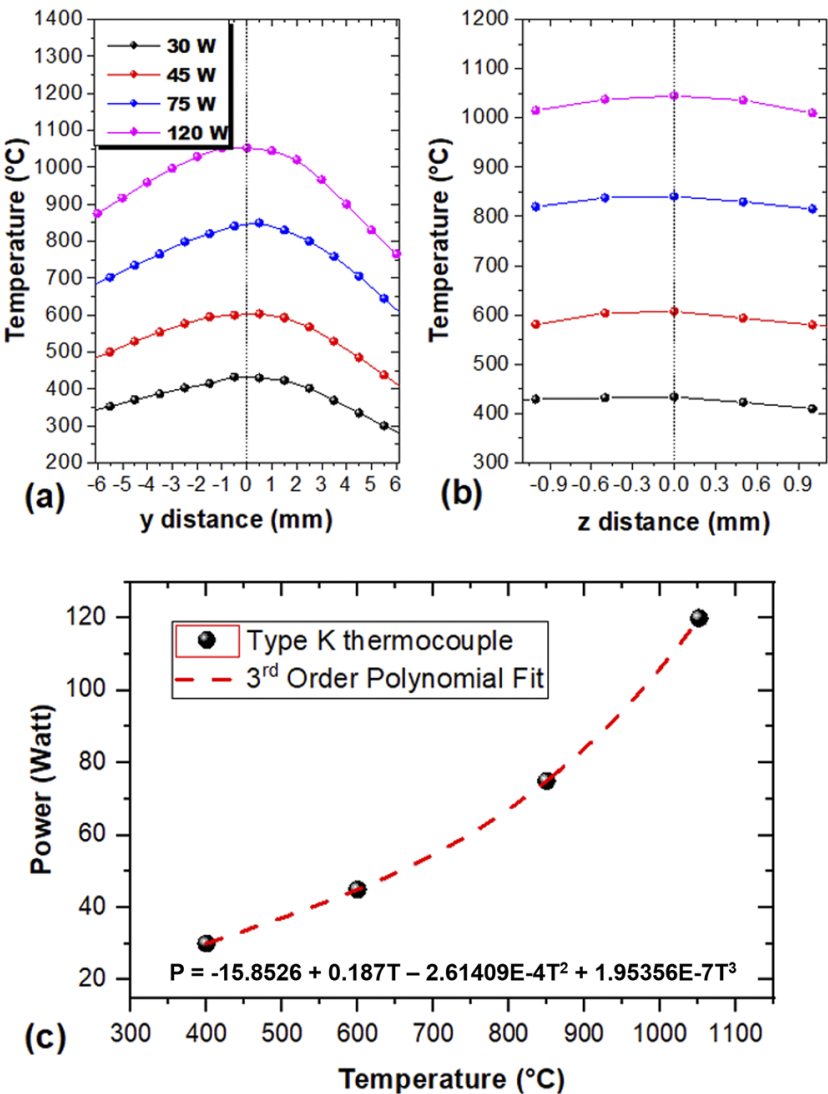

FIG. 3. Spatial temperature distribution at different power settings in (a) $y$ and (b) $z$ directions as measured with a K-type thermocouple. (c) Applied furnace power as a function of temperature. A polynomial fit was used to extrapolate $\approx 1200^{\circ} \mathrm{C}$ at $185 \mathrm{~W}$.

with a K-type thermocouple. The measured temperature increases as a function of power [Fig. 3(c)]; however, the spatial temperature gradient ( $\Delta T$ from the edges to the center) also increases-meaning that the edges of the sample are significantly cooler than the center. Both temperature distributions are expected to be symmetrical around the center; we interpret asymmetry in this case to be caused by misalignment of the lamps. The temperature variation near the sample position is $\leq 9 \mathrm{~K} / \mathrm{mm}^{-1}$ in the $y$ direction and $\leq 2.5 \mathrm{~K} / \mathrm{mm}^{-1}$ in the $z$ direction. The homogeneity in the $z$ direction is particularly important for layer-by-layer scans where a line $\mathrm{x}$-ray beam is used to produce section topography images, which are then assembled into a $3 \mathrm{D}$ model of the sample.

Figure 3(c) shows the power-temperature relationship measured with a K-type thermocouple. A third-order polynomial fit was used to extrapolate $\approx 1200^{\circ} \mathrm{C}$ at $185 \mathrm{~W}$, compatible with most of the industrially relevant heat treatments of steel alloys as they take place well below $1200^{\circ} \mathrm{C}$. We note that higher temperatures could be achieved within the power limits of the lamps, as we did not use the full $400 \mathrm{~W}$ power range for the two halogen lamps in the furnace. For example, we measured a temperature of $1450 \pm 20^{\circ} \mathrm{C}$ at $250 \mathrm{~W}$ using a blackened type-S thermocouple.

\section{B. Characterizing temperature from lattice parameters}

We used a recrystallized grain of $\alpha$ iron within an $\mathrm{Fe}-3 \% \mathrm{Si}$ sample with dimensions of $0.17 \times 0.17 \times 8 \mathrm{~mm}^{3}$ inside a quartz capillary to calibrate the furnace based on thermal expansion within the sample. We measured shifts in the (110) Bragg peak along the vertical direction, using $17 \mathrm{keV}$ x rays that were diffracted onto a $2 \mathrm{D}$ FReLoN CCD detector that was placed $5090 \mathrm{~mm}$ behind the sample. Using the motorized translation stages at ID06, we were able to translate the detector to the appropriate $2 \theta$ angle across the entire temperature range required for the calibration. The $d$-spacing of (110) measured for each furnace power was used to calculate the temperature of the sample using the equation described in Ref. 21. This approach of using the lattice parameter and thermal expansion relation to measure temperature is ideally suited for the $\mathrm{x}$-ray measurements at ID06, so long as care is taken to ensure that the crystal's orientation will diffract at least one beam through the apertures of the furnace (Fig. 4).

\section{Usage during a DFXM experiment}

To demonstrate the utility of the radiation furnace in DFXMbased studies, we include initial data from experiments investigating the evolution of dislocations with temperature (annealing) in singlecrystalline aluminum. The $0.5 \times 0.5 \times 20 \mathrm{~mm}^{3}$ crystals were used as purchased (no further purification or heat treatments) from Surface Preparation Laboratories in this experiment. The samples were cut from a larger ore and polished by chemical etching to mitigate residual surface strains. This experiment performed slow-ramp heating of the samples, with long equilibration times at each temperature, spanning $6 \mathrm{~h}$. The microstructure of the crystal was then measured at a series of temperatures using the dark-field x-ray microscope at the ESRF beamline ID06-HXM. ${ }^{9}$ A Si(111) double crystal monochromator selected $\mathrm{x}$ rays at photon energy $17 \mathrm{keV}$ from the undulator source.

Before reaching the sample, the incident $x$ rays were passed through a $2 \mathrm{D}$ transfocator with compound refractive lenses (CRLs) totaling $8 \mathrm{Be}$ lenslets (an apex radius of curvature $R=200 \mu \mathrm{m}$ ), followed by a $1 \mathrm{D}$ condenser with 58 Be lenslets $(R=200 \mu \mathrm{m})$. A $400 \mu \mathrm{m}$ $\times 600 \mathrm{~nm}$ (horizontal $\times$ vertical) area of the sample was illuminated by the $\mathrm{x}$ rays. The diffracted $\mathrm{x}$ rays were then imaged with an $\mathrm{x}$-ray objective lens comprised of $882 \mathrm{D}$ Be parabolic lenses $(R$ $=50 \mu \mathrm{m}$ ) that was placed $274 \mathrm{~mm}$ behind the sample, producing an effective focal length of $260 \mathrm{~mm}$. A far-field CCD camera was positioned $5364 \mathrm{~mm}$ from the sample to capture the magnified image. The effective magnification was $18.5 \times$, resulting in a final effective pixel size of $75 \mathrm{~nm} /$ pixel when combined with the scintillatorbased optical components used with the detector. The incident x-ray line beam illuminated $\mathrm{a} \approx 600 \mathrm{~nm}$ high layer of the sample to capture section topographs. To map the relative axial strain, longitudinal $(\theta-2 \theta)$ scans were performed by collecting dark-field images across scans of the sample tilt and scans of the objective and camera positions. We probed the (002) Bragg reflection of the aluminum crystal at $2 \theta=20.84^{\circ}$. The temperature was calibrated for the aluminum sample using the thermal expansion coefficients described in Ref. 22.

Figure 5 reveals the refinement of the microstructure with the increase in temperature, covering the temperature range required 

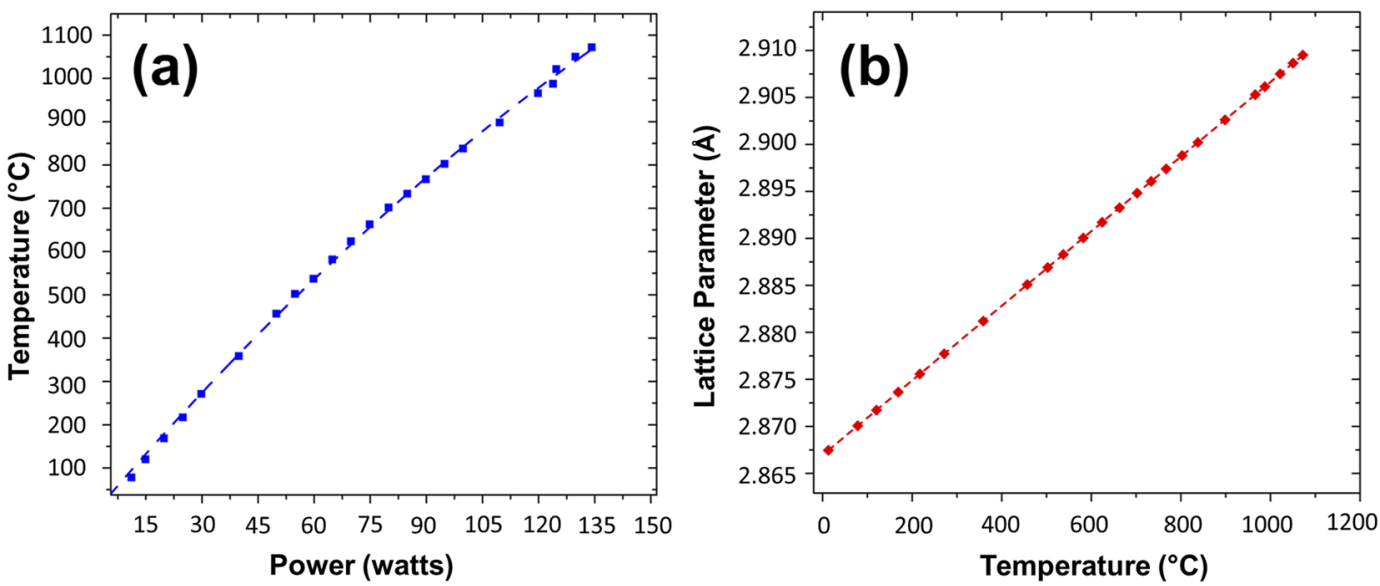

FIG. 4. Temperature calibration curve as measured based on thermal expansion of the lattice parameter in $\alpha$ iron. The (110) Bragg reflection of a grain was traced on a $\mathrm{CCD}$ located $\approx 5 \mathrm{~m}$ downstream of the sample. The plots show (a) the sample temperature as a function of applied power and (b) the measured lattice parameter for each temperature upon heating.
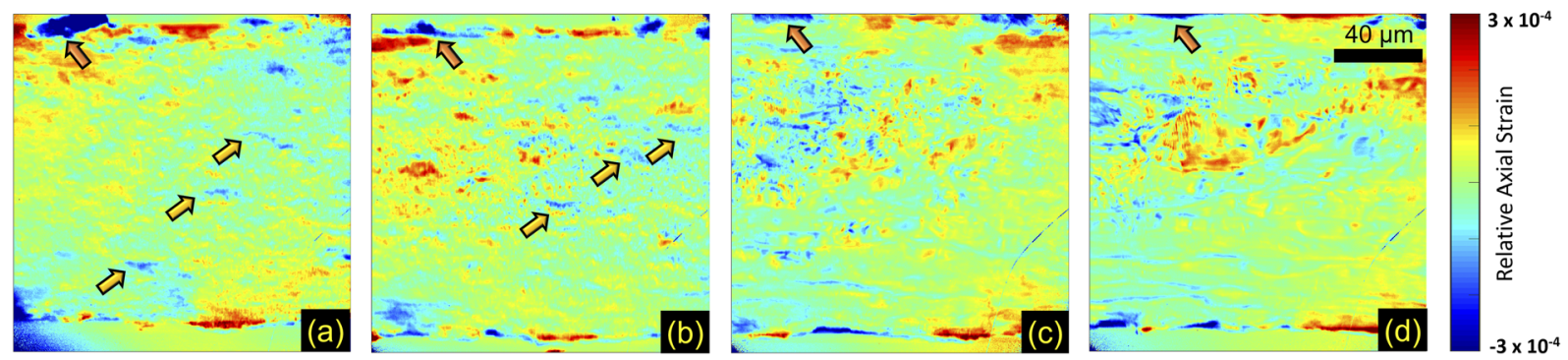

FIG. 5. Reconstructed DFXM relative strain maps of single-crystalline aluminum during in situ isothermal heating at (a) $155^{\circ} \mathrm{C}$ showing mostly positive strain in the interior of the crystal (b) at $252^{\circ} \mathrm{C}$ with some negative strain present in the interior (c) at $416^{\circ} \mathrm{C}$ where the strain fluctuations start to become prominent and linear structures appear (d) at $501^{\circ} \mathrm{C}$ showing higher strain fluctuations with more evident linear structures. The color scale represents relative axial strain.

to anneal. ${ }^{23}$ Arrows over each image point to features that evolve with temperature, which demonstrates that the experiment probed the same region of the sample at each temperature. Analogous to electron backscatter diffraction (EBSD), DFXM resolves local strain and misorientation in crystals that arise from strain or orientation variation across the crystal. With large dislocation densities, the strain measured by DFXM shows the residual strain summed over all lattice distortions within each voxel of our field of the material. With DFXM's $\approx 300 \mathrm{~nm}$ spatial resolution, the changes shown in Fig. 5 display a view of how residual strain fields ${ }^{24}$ evolve during annealing, as the dislocation density reduces from annealing because thermal energy annihilates populations of originally pinned dislocations. ${ }^{25}$ In this case, we see an increase in the residual strain field with the increase in temperature because as fewer dislocations contribute to the residual strain, they cancel each other out to a lesser extent-ultimately reaching higher magnitudes. ${ }^{26}$ The optical furnace in this work enabled the current experiment to probe a large region of sample that is evenly heated with limited thermal gradients. Further studies with this optical furnace may be able to give further insight into the evolution of the microstructure and dislocation density with the increase in temperature in a host of samples.

\section{CONCLUSION}

We demonstrated a radiation furnace developed for DFXM studies at ID06 beamline at the ESRF. The furnace was calibrated up to $1050{ }^{\circ} \mathrm{C}$ using two distinct methods: by means of a K-type thermocouple and by resolving thermal expansion through the lattice parameter in $\alpha$ iron. Extrapolating the calibration curve, we expect that temperatures above $1200^{\circ} \mathrm{C}$ can be reached-potentially higher for samples with very high infrared absorption. This makes the furnace ideal to study steel alloys and ceramics. While originally designed for DFXM studies, this furnace is compatible with the other methods available at ID06 such as bright-field tomography, near-field diffraction topography, and high-resolution reciprocal space mapping. We showed a case study using DFXM of isothermal heating of a pure single-crystal aluminum. Axial strain maps showed subtle structural modifications as a function of temperature, which are characteristic of the annealing that was performed. The limitations of the furnace result from the restricted apertures for the sample and beam. Mosaicity scans are difficult due to the small tilt range orthogonal to the rocking, and full 3DXRD scans are impossible due to the limited size of the beam exit. Nevertheless, these studies are still possible for interrupted 
heating schemes by translating the furnace out without moving the sample.

\section{ACKNOWLEDGMENTS}

We acknowledge the ESRF for provision of beamtime on beamline ID06. We thank Alexis Steinmann of AS Special Devices for his excellent services in the construction of the furnace. The DFXM demonstration of this work was performed under the auspices of the U.S. Department of Energy by Lawrence Livermore National Laboratory under Contract No. DE-AC52-07NA27344 and it was supported, in part, by LLNL, Lawrence Fellowship Program. The authors thank N. Repovz for her detailed proofreading of this manuscript. C.Y. thanks Onderzoeks Centrum voor de Aanwending van Staal (OCAS), Belgium, for the financial support of this project.

\section{DATA AVAILABILITY}

Raw data were generated at the ESRF large scale facility. Derived data supporting the findings of this study are available from the corresponding author upon reasonable request.

\section{REFERENCES}

${ }^{1}$ F. J. Humphreys and M. Hatherly, Recrystallization and Related Annealing Phenomena, 2nd ed. (Elsevier, 2004).

${ }^{2}$ G. Winther, X. Huang, A. Godfrey, and N. Hansen, "Critical comparison of dislocation boundary alignment studied by TEM and EBSD: Technical issues and theoretical consequences," Acta Mater. 52, 4437-4446 (2004).

${ }^{3}$ S. Schmidt, S. F. Nielsen, C. Gundlach, L. Margulies, X. Huang, and D. J. Jensen, "Watching the growth of bulk grains during recrystallization of deformed metals," Science 305, 229-232 (2004).

${ }^{4}$ J. Sun, T. Yu, C. Xu, W. Ludwig, and Y. Zhang, "3D characterization of partially recrystallized Al using high resolution diffraction contrast tomography," Scr. Mater. 157, 72-75 (2018).

${ }^{5}$ H. Simons, A. King, W. Ludwig, C. Detlefs, W. Pantleon, S. Schmidt, F. Stöhr, I. Snigireva, A. Snigirev, and H. F. Poulsen, "Dark-field $\mathrm{x}$-ray microscopy for multiscale structural characterization," Nat. Commun. 6, 6098 (2015).

${ }^{6}$ H. Simons, A. C. Jakobsen, S. R. Ahl, C. Detlefs, and H. F. Poulsen, "Multiscale 3D characterization with dark-field x-ray microscopy," MRS Bull. 41, 454 (2016).

${ }^{7}$ H. F. Poulsen, A. C. Jakobsen, H. Simons, S. R. Ahl, P. K. Cook, and C. Detlefs, "X-ray diffraction microscopy based on refractive optics," J. Appl. Crystallogr. 50, $1441-1456$ (2017)

${ }^{8}$ H. F. Poulsen, P. K. Cook, H. Leemreize, A. F. Pedersen, C. Yildirim, M. Kutsal, A. C. Jakobsen, J. X. Trujillo, J. Ormstrup, and C. Detlefs, "Reciprocal space mapping and strain scanning using $\mathrm{x}$-ray diffraction microscopy," J. Appl. Crystallogr. 51, 1428-1436 (2018).

${ }^{9}$ M. Kutsal, P. Bernard, G. Berruyer, P. K. Cook, R. Hino, A. C. Jakobsen, W. Ludwig, J. Ormstrup, T. Roth, H. Simons, K. Smets, J. X. Sierra, J. Wade,
P. Wattecamps, C. Yildirim, H. F. Poulsen, and C. Detlefs, "The ESRF dark-field x-ray microscope at ID06," IOP Conf. Ser.: Mater. Sci. Eng. 580, 012007 (2019).

${ }^{10}$ D. Fan, W. Zhou, S. Wei, Y. Liu, M. Ma, and H. Xie, "A simple external resistance heating diamond anvil cell and its application for synchrotron radiation x-ray diffraction," Rev. Sci. Instrum. 81, 053903 (2010).

${ }^{11}$ C.-S. Zha and W. A. Bassett, "Internal resistive heating in diamond anvil cell for in situ x-ray diffraction and Raman scattering," Rev. Sci. Instrum. 74, 1255-1262 (2003).

${ }^{12}$ A. Salamat, R. A. Fischer, R. Briggs, M. I. McMahon, and S. Petitgirard, "In situ synchrotron $\mathrm{x}$-ray diffraction in the laser-heated diamond anvil cell: Melting phenomena and synthesis of new materials," Coord. Chem. Rev. 277-278, 15-30 (2014).

${ }^{13}$ R. Boehler, H. Musshoff, R. Ditz, G. Aquilanti, and A. Trapananti, "Portable laser-heating stand for synchrotron applications," Rev. Sci. Instrum. 80, 045103 (2009).

${ }^{14}$ A. Yavari, J. Uriarte, K. Tousimi, A. Le Moulec, W. Botta Filho, K. B. Kim, P. Rejmankova, and Å. Kvick, "In-situ detection of the onset crystallization of $\mathrm{Zr}_{55} \mathrm{Cu}_{30} \mathrm{Al}_{10} \mathrm{Ni}_{5}$ from the bulk glass and the liquid states using synchrotron radiation," Mater. Sci. Forum 307, 17-24 (1999).

${ }^{15} \mathrm{~J}$. Schneider, "Mirror heaters for high temperature x-ray diffraction," Adv. XRay Anal. 36, 397-402 (1992).

${ }^{16}$ P. Sarin, W. Yoon, K. Jurkschat, P. Zschack, and W. M. Kriven, "Quadrupole lamp furnace for high temperature (up to $2050 \mathrm{~K}$ ) synchrotron powder x-ray diffraction studies in air in reflection geometry," Rev. Sci. Instrum. 77, 093906 (2006).

${ }^{17}$ T. Proffen, F. Frey, H. Plöckl, and H. Krane, "A mirror furnace for synchrotron diffraction experiments up to $1600 \mathrm{~K}$," J. Synchrotron Radiat. 2, 229-232 (1995).

${ }^{18}$ A. Bardelcik, C. P. Salisbury, S. Winkler, M. A. Wells, and M. J. Worswick, "Effect of cooling rate on the high strain rate properties of boron steel," Int. J. Impact Eng. 37, 694-702 (2010).

${ }^{19}$ S. Koohpayeh, D. Fort, A. Bradshaw, and J. Abell, "Thermal characterization of an optical floating zone furnace: A direct link with controllable growth parameters," J. Cryst. Growth 311, 2513-2518 (2009).

${ }^{20}$ E. Sparrow, "Radiation heat transfer between surfaces," in Advances in Heat Transfer, edited by J. P. Hartnett and T. F. Irvine (Elsevier, 1965), Vol. 2, pp. 399-452.

${ }^{21}$ Z. S. Basinski, W. Hume-Rothery, and A. Sutton, "The lattice expansion of iron," Proc. R. Soc. London, Ser. A 229, 459-467 (1955).

${ }^{22} \mathrm{~K}$. Wang and R. R. Reeber, "The perfect crystal, thermal vacancies and the thermal expansion coefficient of aluminium," Philos. Mag. A 80, 1629-1643 (2000).

${ }^{23}$ G. K. Williamson and R. E. Smallman, "III. Dislocation densities in some annealed and cold-worked metals from measurements on the x-ray DebyeScherrer spectrum," Philos. Mag. 1, 34-46 (1956).

${ }^{24}$ H. Simons, A. C. Jakobsen, S. R. Ahl, H. F. Poulsen, W. Pantleon, Y.-H. Chu, C. Detlefs, and N. Valanoor, "Nondestructive mapping of long-range dislocation strain fields in an epitaxial complex metal oxide," Nano Lett. 19, 1445-1450 (2019).

${ }^{25} \mathrm{D}$. Hull and D. Bacon, Introduction to Dislocations, 5th ed. (Elsevier Ltd., 2011). ${ }^{26} \mathrm{D}$. Steigmann and R. Ogden, "A note on residual stress, lattice orientation and dislocation density in crystalline solids," J. Elast. 109, 275 (2012). 